\title{
Immunogenetics special issue 2021: Fish Immunology
}

\author{
Johannes M. Dijkstra ${ }^{1} \cdot$ Brian Dixon $^{2}$ \\ Published online: 4 January 2021 \\ (c) The Author(s), under exclusive licence to Springer-Verlag GmbH, DE part of Springer Nature 2021
}

Since 2016, Immunogenetics has published a yearly special issue with articles by leading experts in a particular field. This year, the topic is Fish Immunology. Fish include the most primitive animals with an immune system that is similar to humans, and they provide unique experimental advantages related to breeding. Additionally, fish immunology is of economic relevance to fisheries and aquaculture, the fastest growing agricultural sector globally.

Immunogenetics has a long tradition of reporting fish immunology studies. Jan Klein, one of the journal's two founding fathers - and editor from 1974 to 1997—and two of the current editors, Martin Flajnik and Masanori Kasahara, have made major contributions to the field. The latter two have also co-authored articles in this special issue (see below). In the past, Immunogenetics was the first to report important findings in fish immunology, including for example the first descriptions of classical MHC class I genes in fish (Grimholt et al. 1993) and interleukin 2 (Bird et al. 2005), and an $M h c$ genomic region sequence (Clark et al. 2001). The journal's greatest asset—in a publishing world in which this is increasingly uncommon -is its team of specialized editors and editorial board members. Immunogenetics is probably one of the very few journals where there is a good chance that all reviewers will understand one's submitted paper if it focuses on an immunogenetics topic.

We are grateful to each of the authors because-despite the unusual corona virus-impacted year-they have produced eight exciting papers for this special issue. Unfortunately,

This article is part of Topical Collection on Fish Immunology

Johannes M. Dijkstra

dijkstra@fujita-hu.ac.jp

$\bowtie$ Brian Dixon

bdixon@uwaterloo.ca

1 Institute for Comprehensive Medical Science, Fujita Health University, Dengaku-gakubo 1-98 Toyoake-shi, Aichi-ken 470-1192, Japan

2 Department of Biology, University of Waterloo, 200 University Ave W, Waterloo, ON N2L 3G1, Canada due to the unpredicted circumstances of 2020, several additionally intended manuscripts could not be finished before the deadline. However, we hope that they will appear later in Immunogenetics.

This special issue starts with three articles on the immune systems of primitive fish species: namely, (1) a review on the immune system of jawless fish (Sutoh and Kasahara 2020), (2) a review on the incomplete separation between antibody and $\mathrm{T}$ cell receptor (TCR) genes/genetics in sharks and some other vertebrates (Ott et al. 2020), and (3) an article on how to optimize the detection and alignment of highly diverged sequences in combination with the identification of interleukin $2 / 15 / 15 \mathrm{~L} / 21$ family genes in sharks (Dijkstra 2020). The subsequent five articles are on the immune system of teleost (modern bony) fishes. These include (4) a review on the anatomy of immune structures and organs (Bjørgen and Koppang 2020), (5) a review on antibodies (Bilal et al. 2020), (6) an article on MHC class II in pike and salmonids (Lukacs and Grimholt 2020), (7) an article arguing relatedness between leukocyte immune-type receptors (LITRs) and the Fc-receptor-like family (Wang et al. 2020), and (8) a review on the intersection of stress, sex, and immunity (Campbell et al. 2020).

Sutoh and Kasahara (2020) contributed "The immune system of jawless vertebrates: insights into the prototype of the adaptive immune system." This review on the immune system of jawless fish is a well-written summary of the field, accessible to the novice but nevertheless fairly complete. The adaptive immune system in jawless fish is based on the clonal expression of receptors with leucinerich repeat domains in lymphocytes, assembled at the gene level through somatic recombination. Sutoh and Kasahara highlight the fact that although jawless and jawed vertebrates use a different molecular family for adaptive recognition of antigens, both their innate and adaptive immune systems show important similarities.

Ott, Ohta, Flajnik, and Criscitiello (2020) contributed "Lost structural and functional inter-relationships between Ig and TCR loci in mammals revealed in sharks." This lengthy review is well worth the read, as it describes complicated 
matters in a pleasing manner and in a plain style for the benefit of the reader. The article provides a broad review of $\mathrm{T}$ cell receptor (TCR) and $\mathrm{B}$ cell receptor (BCR, aka immunoglobulin or antibody) genetics in various vertebrates. At the same time, it concentrates on two deviations from the paradigm found in immunology textbooks: namely, in sharks and several other species, some V-gene segments can be used by both TCR and BCR or are part of TCR genes, whereas phylogenetically they correspond to BCR. Furthermore, in sharks and other species, TCR genes can undergo AID-mediated hypermutation, which in human and mouse is only used for BCR.

Dijkstra (2020) contributed "A method for making alignments of related protein sequences that share very little similarity;shark interleukin 2 as an example." This is a method paper that describes how to find and align related but highly diverged sequences. In the words of one of the specialist reviewers, "The manuscript is laid out very clearly and is replete with useful hints and tips for both the experienced and beginners; I would have greatly appreciated this guide when I started my $\mathrm{PhD}$ and I believe that this manuscript should be essential reading for new students to the field of comparative immunogenetics!" To exemplify the method, Dijkstra identified shark interleukin 2 (IL-2) and IL-21 and analyzed them together with shark IL-15 and IL-15-like (IL-15L). The thorough comparison was only possible because of the very recent large accumulation of shark sequences in databases. Peculiar findings were that shark IL-2 appears to have lost the binding motif for IL-15R $\alpha$ (aka IL-2R $\alpha / 15 R \alpha)$ and that shark IL-15 has an even longer and better conserved "leader sequence" than IL-15 in other vertebrates.

Bjørgen and Koppang (2020) contributed a review titled "Anatomy of teleost fish immune structures and organs." Professor Koppang is considered one of the leading experts on the anatomy of teleost fish immune tissues. Whereas many others only search for tissues already known in mammals, or speculate, Koppang has made solid contributions to the field by diligently identifying several previously unknown immune tissues. Besides providing clear descriptions and figures on the anatomy of the teleost fish immune system, the review also contains a critical evaluation of the field. It is a nice article for anyone with an interest in fish immunology, including experienced researchers.

Bilal, Etayo, and Hordvik (2020) contributed "Immunoglobulins in teleosts." It is a clearly written review on the structure, function, and expression of immunoglobulins (antibodies) in different teleost model species. The review covers common features of these molecules, but also highlights the huge differences between teleost species in antibody production, and that overall there appears to be a shift towards "natural antibodies" compared with mammals. Regarding the ongoing discussion of possible specialization of teleost antibody isotypes to mucosal tissues, it is interesting that Bilal and co-workers show in Table $\mathrm{S} 1$ that in almost all investigated cases all three isotypes $\operatorname{IgM}, \operatorname{IgT}$, and $\operatorname{IgD}$ have their highest transcription levels in a teleost peripheral immune tissue such as spleen, head-kidney, or blood.

Lukacs and Grimholt (2020) contributed "Fate of MHCII in salmonids following 4WGD." Around 90 million years ago, an ancestor of salmonid fishes experienced a fourth whole genome duplication (WGD) in the evolution of vertebrates that set this group apart from other teleosts. Northern pike belongs to the fish phylum that forms the sister group of salmonids and did not undergo this 4WGD. Lukacs and Grimholt extensively analyzed MHC class II sequences and genomic regions in Northern pike and seven salmonid species. As well as showing the effects of a WGD event, their findings are in line with previous reports that showed the instability of MHC class II genes in teleosts: namely, even when comparing between these relatively closely related fish species, differential loss of an ancient nonclassical MHC class II lineage and genomic translocations of both classical and nonclassical MHC class II genes were observed. This is a nice article for researchers with an interest in teleost MHC class II or inheritance patterns of gene families.

Wang, Belosevic, and Stafford (2020) wrote "Identification of distinct LRC- and Fc receptor complexlike chromosomal regions in fish supports that teleost leukocyte immune-type receptors are distant relatives of mammalian Fc receptor-like molecules." They investigated the evolution of teleost fish leukocyte immune-type receptors (LITRs), which possess extracellular immunoglobulin superfamily (IgSF) domains and cytoplasmic tails with immunoregulatory motifs. In previous work, they had shown the weak sequence similarity of fish LITR to mammalian Fc receptor-like (FCRL) family molecules, but also some distant relationship to mammalian IgSF molecules encoded in the leukocyte receptor complex (LRC). In the present study, by identifying and analyzing a wider set of sequences, and by also comparing genomic region organizations (gene syntenies), they found additional support for LITRs and FCRLs being more closely related to each other than to LRC molecules. Although the evolution of immunoregulatory IgSF families should probably be considered far from clear, the study by Wang and co-workers is a strong contribution.

Campbell, Dixon, and Whitehouse (2020) contributed "The intersection of stress, sex, and immunity in fishes." As stated by one of the specialist reviewers, this study is a fairly complete overview of the interaction between reproduction, sex, stress, and immunity in fish and is particularly interesting for comparative biologists or aquaculturists. Articles on the topic are conveniently summarized in tables. The article concludes that there are immunological differences between male and female fishes, but that it is hard to distinguish whether that is 
directly caused by hormones or results from differential stresses caused by reproduction strategies.

Taken together, these papers form a broad overview that highlights the diversity of immune responses and strategies in this diverse but ecologically and economically critical group of animals. This special issue will definitely provide the reader with a solid synopsis of the current research challenges and future directions in fish immunology. We hope that readers will find these eight articles as interesting and informative as we do.

Author contributions Both authors contributed equally to this work.

\section{References}

Bilal S, Etayo A, Hordvik I (2020) Immunoglobulins in teleosts. Immunogenetics. https://doi.org/10.1007/s00251-020-01195-1

Bird S, Zou J, Kono T, Sakai M, Dijkstra JM, Secombes C (2005) Characterisation and expression analysis of interleukin 2 (IL-2) and IL-21 homologues in the Japanese pufferfish, Fugurubripes, following their discovery by synteny. Immunogenetics 56:909923. https://doi.org/10.1007/s00251-004-0741-7

Bjørgen H, Koppang EO (2020) Anatomy of teleost fish immune structures and organs. Immunogenetics. https://doi.org/10.1007/ s00251-020-01196-0

Clark MS, Shaw L, Kelly A, Snell P, Elgar G (2001) Characterization of the MHC class I region of the Japanese pufferfish
(Fugurubripes). Immunogenetics 52:174-185. https://doi. org/10.1007/s002510000285

Campbell JH, Dixon B, Whitehouse LM (2020) The intersection of stress, sex, and immunity in fishes. Immunogenetics. https://doi. org/10.1007/s00251-020-01194-2

Dijkstra JM (2020) A method for making alignments of related protein sequences that share very little similarity; shark IL-2 as an example. Immunogenetics. https://doi.org/10.1007/s00251-02001191-5

Grimholt U, Hordvik I, Fosse VM, Olsaker I, Endresen C, Lie O (1993) Molecular cloning of major histocompatibility complex class I cDNAs from Atlantic salmon (Salmosalar). Immunogenetics 37:469-473. https://doi.org/10.1007/BF00222473

Lukacs M, Grimholt U (2020) Fate of MHCII in salmonids following 4WGD. Immunogenetics. https://doi.org/10.1007/s00251-02001190-6

Ott J, Ohta Y, Flajnik MF, Criscitiello MF (2020) Lost structural and functional inter-relationships between Ig and TCR loci in mammals revealed in sharks. Immunogenetics. https://doi. org/10.1007/s00251-020-01183-5

Sutoh Y, Kasahara M (2020) The immune system of jawless vertebrates: insights into the prototype of the adaptive immune system. Immunogenetics. https://doi.org/10.1007/s00251-02001182-6

Wang J, Belosevic M, Stafford JL (2020) Identification of distinct LRC- and Fc receptor complex-like chromosomal regions in fish supports that teleost leukocyte immune-type receptors are distant relatives of mammalian Fc receptor-like molecules. Immunogenetics. https://doi.org/10.1007/s00251-020-01193-3

Publisher's Note Springer Nature remains neutral with regard to jurisdictional claims in published maps and institutional affiliations. 